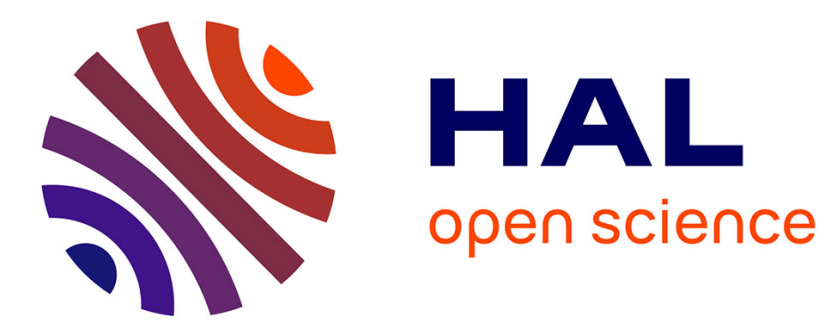

\title{
Energetics of active fluctuations in living cell
}

Étienne Fodor, Kiyoshi Kanazawa, Hisao Hayakawa, Paolo Visco, Frédéric van Wijland

\section{To cite this version:}

Étienne Fodor, Kiyoshi Kanazawa, Hisao Hayakawa, Paolo Visco, Frédéric van Wijland. Energetics of active fluctuations in living cell. Physical Review E: Statistical, Nonlinear, and Soft Matter Physics, 2014, 90, pp.42724. 10.1103/PhysRevE.90.042724 . hal-01081354

\section{HAL Id: hal-01081354 https://hal.science/hal-01081354}

Submitted on 7 Nov 2014

HAL is a multi-disciplinary open access archive for the deposit and dissemination of scientific research documents, whether they are published or not. The documents may come from teaching and research institutions in France or abroad, or from public or private research centers.
L'archive ouverte pluridisciplinaire HAL, est destinée au dépôt et à la diffusion de documents scientifiques de niveau recherche, publiés ou non, émanant des établissements d'enseignement et de recherche français ou étrangers, des laboratoires publics ou privés. 


\title{
Energetics of active fluctuations in living cells
}

\author{
É. Fodor, ${ }^{1,}{ }^{*}$ K. Kanazawa,,${ }^{2}$ H. Hayakawa, ${ }^{2}$ P. Visco, ${ }^{1}$ and F. van Wijland ${ }^{1}$ \\ ${ }^{1}$ Laboratoire Matière et Systèmes Complexes, UMR 7057 CNRS/P7, Université Paris Diderot, \\ 10 rue Alice Domon et Léonie Duquet, 75205 Paris cedex 13, France \\ ${ }^{2}$ Yukawa Institute for Theoretical Physics, Kyoto University, Kitashirakawa-oiwake cho, Sakyo-ku, Kyoto 606-8502, Japan
}

(Received 10 June 2014; published 29 October 2014)

\begin{abstract}
The nonequilibrium activity taking place in a living cell can be monitored with a tracer embedded in the medium. While microrheology experiments based on optical manipulation of such probes have become increasingly standard, we put forward a number of experiments with alternative protocols that, we claim, will provide insight into the energetics of active fluctuations. These are based on either performing thermodynamiclike cycles in control-parameter space or determining response to external perturbations of the confining trap beyond simple translation. We illustrate our proposals on an active itinerant Brownian oscillator modeling the dynamics of a probe embedded in a living medium.
\end{abstract}

DOI: 10.1103/PhysRevE.90.042724

PACS number(s): 87.10.Mn, 87.15.A-, 05.40.-a

\section{INTRODUCTION}

A living cell is a nonequilibrium system which needs to constantly maintain its activity to preserve an organized structure. Major contributors to this activity are the molecular motors which generate forces of the order of a piconewton within the cell. This force generation is an essential process for life as it is the basis of cell motility, wound healing, and cell division. It is fueled by adenosine triphosphate hydrolysis, thus being a nonequilibrium process commonly named an active process. The force is applied by the motors on some polar self-assembled filaments, such as the actin filaments for myosin motors. The polarity of these filaments added to the force generation enable the motors to perform a stochastic directed motion. These phenomena have been experimentally explored in vivo with living cells $[1,2]$ and in vitro, with reconstituted actin gels in which molecular motors density can be externally controlled $[3,4]$.

One of the major experimental techniques which has uncovered the nonequilibrium behavior of living cells and active gels is microrheology [2-6]. Thanks to the progress of high resolution microscopy, it is now possible to track micron sized probes injected into complex fluids, including living organisms. In addition, by means of optical or magnetic tweezers, one can apply a controlled force on these probes and measure rheological properties such as complex shear modulus [7-9] or creep function [10]. By combining these two measurements, it has been possible to quantify the extent to which the fluctuation-dissipation theorem (FDT) is violated these systems [5,11]. So far, the central quantity that has been investigated is a frequency-dependent effective temperature [12-16], which serves as an all-purpose measurement of the distance from thermal equilibrium.

Our aim in this paper is to put forward other quantities that can reveal interesting properties of nonequilibrium activity and that can be measured with the same experimental toolbox of microrheology. In order to render the presentation of these methods more concrete, their predictions are illustrated on a

*Corresponding author: etienne.fodor@ univ-paris-diderot.fr recent theoretical model [1] describing the dynamics of a probe in an active medium.

We begin with giving the basic physical ingredients of our model in Sec. II. We then discuss the simplest protocols in which the spring constant of a harmonic external potential is changed with time in Sec. III. In Sec. IV, we use a quartic potential for which two parameters are changed in time to mimic a thermodynamic cycle [17]. In Sec. V, we review an already proposed method of extracting correlations between active force and position [18] by exploiting the extended fluctuation-dissipation relations [19]. In Sec. VI, we apply the Harada-Sasa relation to quantify the dissipation rate arising from the nonequilibrium behavior of the probe [20].

\section{MODEL}

We model the dynamics of the tracer's position $\mathbf{r}$ by means of an overdamped Langevin equation as described in [1]. From a physical viewpoint, the active medium has a complex polymer cross-linked reticulated structure, surrounded by a viscous Newtonian fluid. The complex structure of the network confines the particle, and we model this as a harmonic potential acting on the probe, centered at position $\mathbf{r}_{0}$. Active forces which originate from surrounding molecular motors continuously modify the network structure, thus spatially translating the minimum of this potential. However, the bead itself modifies the internal network dynamics: Arbitrarily large local deformations are unlikely. To account for this feedback mechanism, we introduce a small backaction force on the potential location. Since the harmonic trap models the confinement by the network, the characteristic size of the trap is much larger than the particle size to avoid any escape of the particle as shown in Fig. 1(a). The backaction force is then necessarily small compared to the force applied on the particle, a feature which we will have to verify in actual experiments. In other words, the tracer dynamics has only a small effect on the $\mathbf{r}_{0}$ dynamics, and was, in fact, neglected in [1]. Moreover, the thermal fluctuations applied on the potential center position $\mathbf{r}_{0}$ must be taken into account, and the corresponding fluctuation amplitude should be negligible compared with the ones of thermal force applied on the tracers. Introducing a dimensionless parameter $\varepsilon \ll 1$, which, 


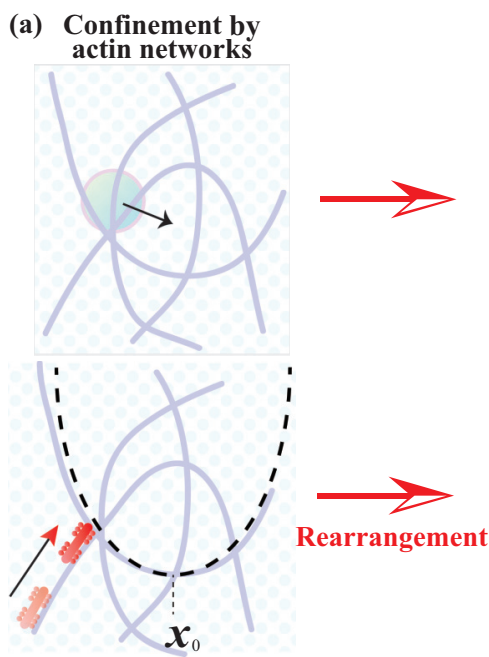

Motion of myosin

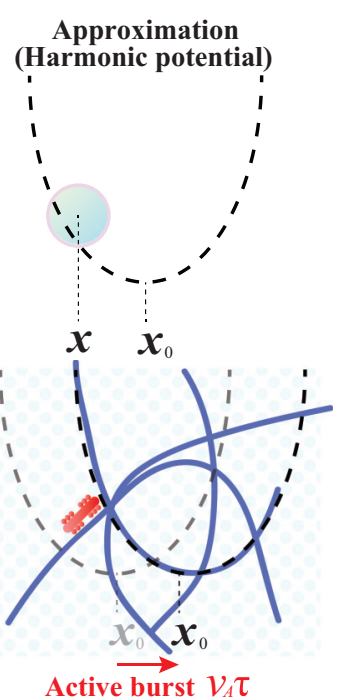

Active burst $\mathcal{V}_{A} \tau$

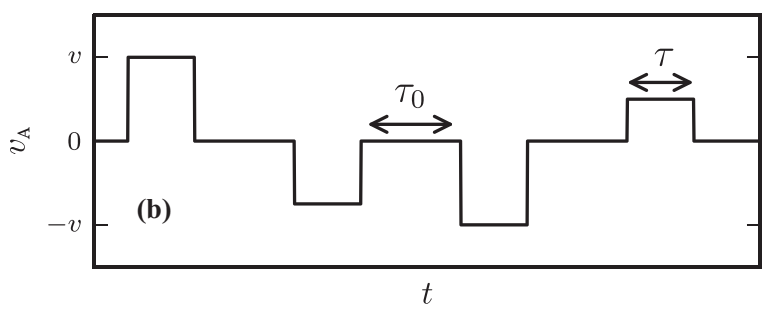

FIG. 1. (Color online) (a) Schematic representation of the energetic landscape rearrangement due to motor activity and its modeling using the active burst applied on the local minimum. In the passive case without motors, the tracer is confined within a harmonic potential. When motors are introduced, their activity modifies the network structure, thus leading to a displacement $v_{\mathrm{A}} \tau$ of the potential local minimum $x_{0}$. (b) Example trajectory of the active burst projection $v_{\mathrm{A}}$. It equals zero over a random duration of average $\tau_{0}$ and is a random value between $-v$ and $v$ during a random time of $\operatorname{order} \tau$.

we anticipate, will be small, we arrive at the coupled set of equations

$$
\begin{aligned}
\frac{d \mathbf{r}}{d t} & =-\frac{1}{\tau_{\mathrm{d}}}\left(\mathbf{r}-\mathbf{r}_{0}\right)+\sqrt{2 D_{\mathrm{T}}} \boldsymbol{\xi}, \\
\frac{d \mathbf{r}_{0}}{d t} & =-\frac{\varepsilon}{\tau_{\mathrm{d}}}\left(\mathbf{r}_{0}-\mathbf{r}\right)+\mathbf{v}_{\mathrm{A}}+\sqrt{2 \varepsilon D_{\mathrm{T}}} \boldsymbol{\xi}_{0},
\end{aligned}
$$

where $T$ is the bath temperature, $\gamma$ is the friction coefficient of the tracer particle with the surrounding environment, $k$ is the spring constant of the harmonic trap, $D_{\mathrm{T}}=T / \gamma$ is a thermal diffusion coefficient, and $\tau_{\mathrm{d}}=\gamma / k$ is a microscopic time scale. The Gaussian white noises $\boldsymbol{\xi}$ and $\boldsymbol{\xi}_{0}$ accounting for thermal fluctuations are uncorrelated, and $\mathbf{v}_{\mathrm{A}}$ is another noise term, referred to as an active burst, describing the effect of molecular motors on the network structure. It denotes the velocity at which the potential is moving, and we model it as a stochastic process inspired from the dynamics of individual motors: There are quiescent periods of random duration of average time $\tau_{0}$ alternating with active bursts of typical velocity $v$ in a random direction and for a random time of average $\tau$. In the absence of active forces, this is the itinerant oscillator model

introduced by Hill [21] and Sears [22] within the framework of simple liquids dynamics (see [23] for a review) which has equilibrium dynamics. Such dynamics for the tracer particles is associated with a complex modulus of the form [24,25]

$$
G^{*}(\omega)=i \omega \eta \frac{1+\varepsilon+i \omega \tau_{\mathrm{d}}}{\varepsilon+i \omega \tau_{\mathrm{d}}}
$$

The viscosity $\eta$ is related to the friction coefficient $\gamma$ via Stokes' law: $\gamma=6 \pi a \eta$, where $a$ is the tracers' radius. Within this minimal rheology, we assume the material behaves like a fluid at short and large time scales, with associated viscosity $\eta$ and $\eta / \varepsilon$, respectively, to leading order in $\varepsilon$. Thus, this material behaves like a much more viscous fluid at large time scale compared with the short time scale behavior. In experimental measurements, one has direct access to one dimensional projections of the position. We shall thus look at the one dimensional projection of Eq. (1) on the scalar position $x$,

$$
\begin{aligned}
\frac{d x}{d t} & =-\frac{1}{\tau_{\mathrm{d}}}\left(x-x_{0}\right)+\sqrt{2 D_{\mathrm{T}}} \xi \\
\frac{d x_{0}}{d t} & =-\frac{\varepsilon}{\tau_{\mathrm{d}}}\left(x_{0}-x\right)+v_{\mathrm{A}}+\sqrt{2 \varepsilon D_{\mathrm{T}}} \xi_{0},
\end{aligned}
$$

where $\left\langle\xi(t) \xi\left(t^{\prime}\right)\right\rangle=\delta\left(t-t^{\prime}\right)=\left\langle\xi_{0}(t) \xi_{0}\left(t^{\prime}\right)\right\rangle$ are still Gaussian noises, and $v_{\mathrm{A}}$ equals 0 over a random duration of order $\tau_{0}$ and is a uniform random value between $-v$ and $v$ over the duration of average $\tau$, as depicted in Fig. 1(b). The active burst projection $v_{\mathrm{A}}$ is a non-Gaussian process [26,27], and the two-time correlation function reads $\left\langle v_{\mathrm{A}}(t) v_{\mathrm{A}}(0)\right\rangle=$ $T_{\mathrm{A}} e^{-|t| / \tau} /(\tau \gamma)$. The energy scale $T_{\mathrm{A}}$ defines an effective active temperature in terms of the duty ratio $p_{\mathrm{on}}=\tau /\left(\tau+\tau_{0}\right)$ :

$$
T_{\mathrm{A}}=\frac{\gamma v^{2} \tau p_{\mathrm{on}}}{3}
$$

This temperature is frequency independent, and we describe in this paper several methods to measure this quantity. It quantifies the amplitude of the active fluctuations, as defined by the active force correlations, and we will see later that it characterizes the tracer's statistics at large time scale. We postpone to Appendix A the derivation of the $n$-time correlation function of the active burst $v_{\mathrm{A}}$. We derive the analytic expressions of the physical observables to leading order in $\varepsilon$.

To describe the phenomenology of this model, we focus on the mean square displacement (MSD) $\left\langle\Delta x^{2}\right\rangle\left(t_{\mathrm{i}}, t_{\mathrm{f}}\right)=$ $\left\langle\left(x\left(t_{\mathrm{i}}\right)-x\left(t_{\mathrm{f}}\right)\right)^{2}\right\rangle$. Even though the MSD depends on two time variables, in the limit where the initial time $t_{\mathrm{i}}$ is large enough compared to the microscopic relaxation time scale $\tau_{\mathrm{d}}$ it becomes effectively a function of the only time lag $t=t_{\mathrm{f}}-t_{\mathrm{i}}$. This is the case we consider in this paper, as we only consider quasistatic transformations. Using the Fourier transform of Eq. (3), we compute the position autocorrelation function $C(t)=\langle x(t) x(0)\rangle$, from which we deduce the MSD as $\left\langle\Delta x^{2}\right\rangle(t)=2[C(0)-C(t)]$. We denote the thermal contribution to the MSD by $\left\langle\Delta x_{\mathrm{T}}^{2}\right\rangle$ and the MSD when the particle is only subjected to motor activity by $\left\langle\Delta x_{\mathrm{A}}^{2}\right\rangle$. We compute these 
two contributions to leading order in $\varepsilon$ :

$$
\begin{aligned}
\left\langle\Delta x_{\mathrm{T}}^{2}\right\rangle(t)= & \frac{2 T}{k}\left(1-e^{-t / \tau_{\mathrm{d}}}+\varepsilon \frac{t}{\tau_{\mathrm{d}}}\right), \\
\left\langle\Delta x_{\mathrm{A}}^{2}\right\rangle(t)= & \frac{2 T_{\mathrm{A}} / k}{1-\left(\tau / \tau_{\mathrm{d}}\right)^{2}}\left[e^{-t / \tau_{\mathrm{d}}}+\frac{t}{\tau_{\mathrm{d}}}-1\right. \\
& \left.+\left(\frac{\tau}{\tau_{\mathrm{d}}}\right)^{3}\left(1-e^{-t / \tau}-\frac{t}{\tau}\right)\right] .
\end{aligned}
$$

The expression without any assumption made on $\varepsilon$ is presented in Appendix B 1. In the active case, the time evolution of the MSD exhibits a two step growth with an intermediate plateau. The first growth and saturation correspond to the equilibriumlike behavior of a probe caged in a fixed trap. The initial growth is diffusive with a standard diffusion coefficient $D_{\mathrm{T}}$, and the plateau value is given by $2 T / k$. The evolution of the MSD at larger time scales, reflecting the nonequilibrium features of the system, is a diffusive growth with a diffusion coefficient $\varepsilon D_{\mathrm{T}}+D_{\mathrm{A}}$, where $D_{\mathrm{A}}=T_{\mathrm{A}} / \gamma$ is an "active" diffusion coefficient. In the passive case, the tracer particle can also escape the confinement at time scales larger $\tau_{\mathrm{d}} / \varepsilon$, and the large time scale diffusion coefficient $\varepsilon D_{\mathrm{T}}$ is small compared to the short time scale one, as shown in Fig. 2(a).

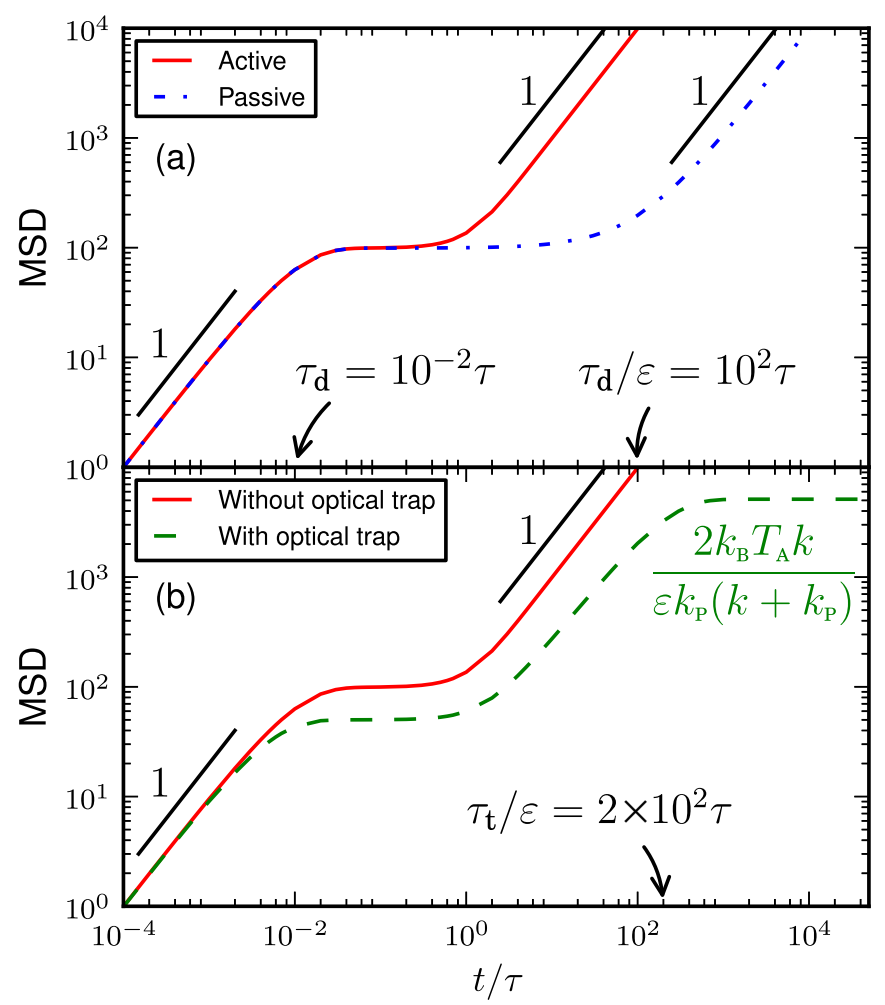

FIG. 2. (Color online) (a) Mean square displacement as a function of the scaled time $t / \tau$ for active (red solid line) and passive (blue dot-dashed line) systems. (b) Mean square displacement as a function of the scaled time $t / \tau$ with (green dashed line) and without (red solid line) an external potential, in this case a harmonic optical trap. The evolution is qualitatively similar for time scale smaller than $\tau_{\mathrm{t}} / \varepsilon$. At large time scale, there is a plateau due to the confinement within the optical trap, which value diverges with $\varepsilon$. $\left\{T, \varepsilon, k, \tau_{\mathrm{d}}, \tau_{\mathrm{t}}, T_{\mathrm{A}}, \tau\right\}=\left\{10^{2}, 10^{-4}, 2,10^{-2}, 2 \times 10^{2}, 1,1\right\}$.
The expression of the thermal diffusion coefficient at large time scale agrees with the fluidlike behavior of the material with the associated viscosity $\eta / \varepsilon$. The backaction reflects the ability of the particle to modify its environment. The local minimum motion is not only affected by activity within the network, but also by the interaction of the bead with the network. The large time scale diffusion in the passive case is in agreement with experimental observations of tracers embedded in living cells $[1,3]$. Assuming $\tau_{\mathrm{d}} \simeq 1 \mathrm{~ms}$ and given that a large time scale diffusion appears for $t>10 \mathrm{~s}$ in [1], we deduce $\varepsilon \simeq 10^{-4}$ in agreement with $\varepsilon \ll 1$.

\section{VARYING THE SPRING CONSTANT}

One of the most fruitful approaches to gather information in living cells has been achieved by applying external forces to probe particles. This has been carried out by different methods, such as optical or magnetic tweezers [5,28], resulting in an effective external potential $U_{\mathrm{P}}$ acting on the probe. To our knowledge, the general protocol has always been to apply the potential and then to execute a space translation, typically with an oscillation, to measure quantities such as the complex shear modulus. Here we would like to pursue a different route, where, instead of translating the potential well, we consider a timedependent change in other parameters of the external potential. Our main goal is to design a protocol with time-dependent parameters and to estimate the work extracted over the whole protocol. Optical tweezers effects are well approximated by a harmonic potential, though more complex energy landscape can be crafted [29].

The simplest protocol is thus to slowly vary the spring constant $k_{\mathrm{P}}$ in time (this is sometimes called a "capture experiment" [30]). We consider that an external potential $U_{\mathrm{P}}=k_{\mathrm{P}} x^{2} / 2$ is applied to the tracer as presented in Fig. 3, so that an additional term $-k_{\mathrm{P}} x / \gamma$ is to be inserted in the $x$ dynamics in Eq. (3a). We postpone the derivation of the MSD to Appendix B 2. Within our model, when we apply this external force, the evolution of the MSD for time scales smaller than $\tau_{\mathrm{t}} / \varepsilon$, where $\tau_{\mathrm{t}}=\gamma\left(k+k_{\mathrm{P}}\right) /\left(k k_{\mathrm{P}}\right)$ to leading order in $\varepsilon$, is qualitatively similar to the case without optical trap. At large time scale, the MSD saturates, meaning the tracer is confined within the optical trap. After a relaxation time $\tau_{\mathrm{t}} / \varepsilon$, the system reaches a steady state characterized by active fluctuations, the optical trap stiffness, and the properties of the network via $k$ as presented in Fig. 2(b). Note that the plateau value

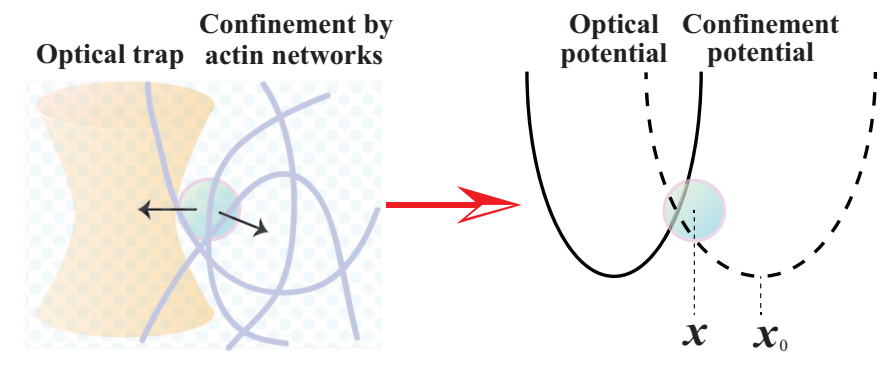

FIG. 3. (Color online) Schematic representation of the energetic landscape when a quadratic optical trap is applied on the tracers, in addition to the harmonic confinement potential. 
$2 T_{\mathrm{A}} k /\left[\varepsilon k_{\mathrm{P}}\left(k+k_{\mathrm{P}}\right)\right]$ does not depend on the bath temperature $T$ to leading order in $\varepsilon$, and it diverges with $\varepsilon$ so that the backaction is necessary to model the confinement of the bead by the optical trap. We show that the stationary displacement probability density function is a Gaussian distribution to leading order in $\varepsilon$, so that the non-Gaussian nature of the active process $v_{\mathrm{A}}$ does not affect the steady-state tracer's distribution to that order of the calculation. Likewise, the leading term in $\varepsilon$ of the tracer's stationary distribution is unchanged when considering a white noise for $v_{\mathrm{A}}$, be it Gaussian or not. The time scales $\tau$ and $\tau_{0}$ do appear to the next orders in $\varepsilon$ of the steady-state distribution, though. To quantify the deviation of the stationary distribution from a Gaussian distribution, we determine the non-Gaussian parameter (NGP),

$$
\kappa=\frac{\left\langle x^{4}\right\rangle_{\mathrm{SS}}}{3\left\langle x^{2}\right\rangle_{\mathrm{SS}}^{2}}-1
$$

where $\langle\cdot\rangle_{\text {SS }}$ denotes the steady-state average. The NGP is zero for a Gaussian distribution and is often used to quantify deviations to the Gaussian distribution [31]. We compute this quantity to leading order in $\varepsilon$, as presented in Appendix B 2:

$$
\kappa=\frac{2 \varepsilon}{5\left(1+k / k_{\mathrm{P}}\right)} \frac{9 \tau_{0}^{2}+3 \tau \tau_{0}-\tau^{2}}{\left(\tau+\tau_{0}\right) \tau_{\mathrm{d}}} .
$$

The NGP is proportional to $\varepsilon$, as another evidence that the tracer's statistics is Gaussian to leading order in $\varepsilon$. As far as the active temperature $T_{\mathrm{A}}$ is concerned, it can be determined independently of the active time scales by applying a quadratic external potential on the tracer and by measuring its stationary distribution of displacement. It can also be measured from the large time scale diffusion in the absence of external potential. The method we propose is more convenient because the tracer does not experience large excursions, which would otherwise make it hard to keep in focus, as it remains confined within the optical trap.

The backaction reflects the ability of the tracer to act on the surrounding network, thus affecting the dynamics of the local minimum. In the present case, it exerts a force on the network which compensates the driving force due to the active burst, so that a work is applied by the tracer on the network. We see that the measurement of this work enables one to characterize activity within the system. We consider a protocol where $k_{\mathrm{P}}$ is slowly varied from $k_{\mathrm{i}}$ to $k_{\mathrm{f}}$; that is, the time evolution of the protocol is much longer than $\tau_{\mathrm{t}} / \varepsilon$ and the time variation of $k_{\mathrm{P}}$ is negligible compared to the inverse duration of the protocol in terms of $\varepsilon$. The average quasistatic work $W_{\mathrm{H}}$ done by applying the external potential to the probe is $[32,33]$

$$
W_{\mathrm{H}}=\frac{1}{2} \int d k_{\mathrm{P}}\left\langle x^{2}\right\rangle_{\mathrm{SS}},
$$

where the $\langle\cdot\rangle_{\mathrm{SS}}$ means that the average is taken in the steady state with a fixed optical trap, in the present case a harmonic trap of constant $k_{\mathrm{P}}$. We determine the expression of this quasistatic work in Appendix B 2. It takes the form

$$
\begin{aligned}
W_{\mathrm{H}}=E_{\mathrm{H}}\left(k_{\mathrm{f}}\right) & -E_{\mathrm{H}}\left(k_{\mathrm{i}}\right), \text { where } E_{\mathrm{H}} \text { reads } \\
E_{\mathrm{H}}\left(k_{\mathrm{P}}\right)= & \frac{T_{\mathrm{A}}}{2 \varepsilon} \ln \left[\frac{k_{\mathrm{P}}}{k+k_{\mathrm{P}}}\right]-\frac{k T_{\mathrm{A}}}{2\left(k+k_{\mathrm{P}}\right)}+\frac{T}{2} \ln \left[k_{\mathrm{P}}\right] \\
& +\frac{T_{\mathrm{A}}}{2}\left(\frac{\tau}{\tau_{\mathrm{d}}}\right)^{2} \ln \left[\frac{k_{\mathrm{P}} \tau+k\left(\tau+\tau_{\mathrm{d}}\right)}{k+k_{\mathrm{P}}}\right] \\
& -\frac{T_{\mathrm{A}}}{2} \ln \left[\frac{k_{\mathrm{P}}}{k+k_{\mathrm{P}}}\right]+O(\varepsilon) .
\end{aligned}
$$

This energy scale is defined up to a constant which should render the argument of the logarithms dimensionless. It diverges with $\varepsilon$, meaning that if the backaction mechanism were neglected it would take an infinite work to confine the tracer in a harmonic well. This result does not depend on the non-Gaussian nature of the active noise, since $W_{\mathrm{H}}$ is only affected by the second moment of the tracers' statistics, and the dynamics of the tracer's position $x$ is linear in $x$. We have run numerical simulations to determine the accuracy of the above formula. There is perfect agreement with our prediction for small values of $\varepsilon$. When $\varepsilon \simeq 0.15$, the term of order $\varepsilon$ in Eq. (9) is no longer negligible. We compute the expression of the $O(\varepsilon)$ correction term, and we show it indeed explains for the deviation of numerical results with Eq. (9), as presented in Fig. 4. Note that in the passive case, without active bursts, the work does not vanish but reduces to the difference of the Helmholtz free energies, as it should for an isothermal transformation. This contribution enters in the $O(1)$ term of the above formula. An interesting feature of formula (9) is that the work is independent of $T$ to leading order in $\varepsilon$, meaning that it should be possible to directly access $T_{\mathrm{A}}$ with a rather simple protocol. For example, one could measure the average

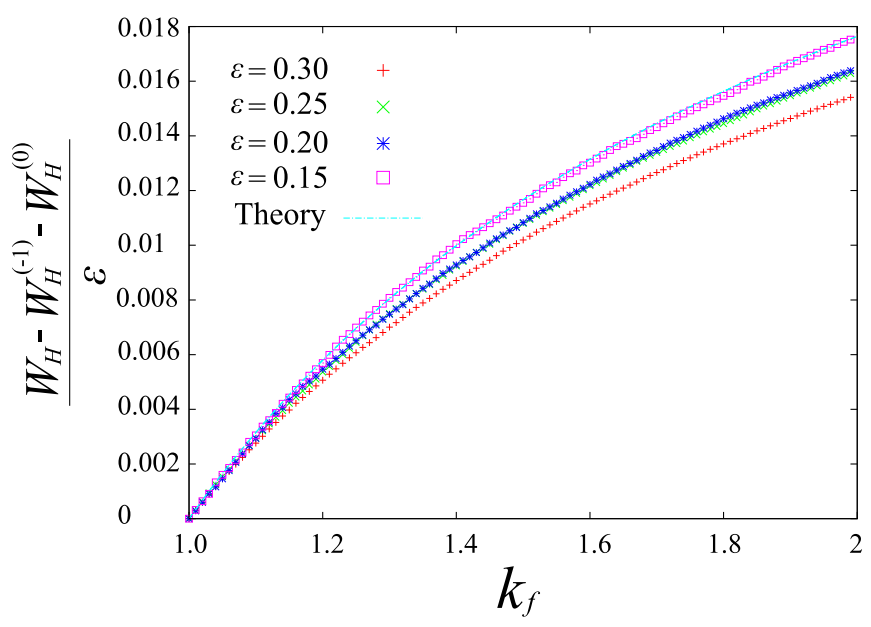

FIG. 4. (Color online) Study of the influence of the $O(\varepsilon)$ correction term in Eq. (9). The quasistatic work $W_{\mathrm{H}}$ is obtained numerically from simulations of the dynamics in Eq. (3), where $\varepsilon=\{0.3,0.25,0.2,0.15\}$. We extract the correction term as $W_{\mathrm{H}}-$ $W_{\mathrm{H}}^{(-1)}-W_{\mathrm{H}}^{(0)}$, where the expression of $W_{\mathrm{H}}^{(n)}=O\left(\varepsilon^{n}\right)$ is given by Eq. (9). The analytic expression of the $O(\varepsilon)$ correction term is plotted in a cyan dotted line as a function of $k_{\mathrm{f}}$, and it agrees with numerical simulations for $\varepsilon=0.15$. For larger values of $\varepsilon$, the next order terms should be taken into account to explain the deviation of the simulated quasistatic work from the prediction in Eq. (9). $\left\{T, k, k_{\mathrm{i}}, \gamma, \tau_{0}, \tau, v\right\}=\{0,1,1,1,5,0.6,4\}$. 
work with different values of $k_{\mathrm{i}}$ and $k_{\mathrm{f}}$ to deduce values for $k, \varepsilon$, and $T_{\mathrm{A}}$. However, one should be aware that the protocol has to be operated over large time scales to remain quasistatic. If the operator reduces the volume accessible by the bead by setting $k_{\mathrm{f}}>k_{\mathrm{i}}$, the work is positive, in agreement with the fact that the probe "cools down" when $k_{\mathrm{P}}$ increases. Considering a circular protocol for which $k_{\mathrm{f}}=k_{\mathrm{i}}$, the extracted work is zero as for an equilibrium process. The nonequilibrium properties remain hidden for a circular protocol when a harmonic trap is applied to a tracer.

\section{THERMODYNAMIC CYCLES WITH QUARTIC POTENTIALS}

By combining multiple optical tweezers it is possible to confine the tracer in a more complex potential such as a double well [29]. The corresponding quartic optical trap $U_{\mathrm{P}}=k_{\mathrm{P}} x^{2} / 2+b_{\mathrm{P}} x^{4} / 4$ depends on two parameters that are both tunable by the operator. In particular, the parameter $k_{\mathrm{P}}$ can take negative values, as long as the condition $k+k_{\mathrm{P}}>0$ is fulfilled. We regard the potential anharmonicity as a small perturbation with respect to the harmonic case: $b_{\mathrm{P}}=O\left(\varepsilon^{n}\right)$. Our picture is that $\varepsilon$ is a material-dependent quantity, but the shape of the trap, namely the parameter $n$, is fully controlled by the operator. We consider a quasistatic protocol where $k_{\mathrm{P}}$ varies as before and $b_{\mathrm{P}}$ is set constant. The associated work is expressed as $W_{\mathrm{Q}}=\int d k_{\mathrm{P}}\left\langle x^{2}\right\rangle_{\mathrm{SS}} / 2$. The steady-state average is different from the value presented before due to the quartic term in the optical trap. By using a perturbation method with respect to $b_{\mathrm{P}}$, we derive the expression of this steady-state average to order $b_{\mathrm{P}}$. It follows that the average quasistatic work from an initial value $k_{\mathrm{i}}$ to a final one $k_{\mathrm{f}}$ is expressed as $W_{\mathrm{Q}}=W_{\mathrm{H}}+E_{\mathrm{Q} 1}\left(k_{\mathrm{f}}, b_{\mathrm{P}}\right)-E_{\mathrm{Q} 1}\left(k_{\mathrm{i}}, b_{\mathrm{P}}\right)+O\left(b_{\mathrm{P}}^{2}\right)$, where $E_{\mathrm{Q} 1}$ is linear in $b_{\mathrm{P}}$. We compute the expression of $E_{\mathrm{Q} 1}$ to leading order in $\varepsilon$, as presented in Appendix B 3:

$$
\begin{aligned}
\frac{E_{\mathrm{Q} 1}\left(k_{\mathrm{P}}, b_{\mathrm{P}}\right)}{b_{\mathrm{P}}}= & \left(\frac{T_{\mathrm{A}}}{2 k \varepsilon}\right)^{2}\left\{\frac{2 k^{4}}{k_{\mathrm{P}}^{2}\left(k+k_{\mathrm{P}}\right)^{2}}+\frac{3 k^{2} \tau}{k_{\mathrm{P}}^{2}\left(\tau+\tau_{\mathrm{d}}\right)}\right. \\
& -6 k \tau\left[\frac{3 \tau+2 \tau_{\mathrm{d}}}{k_{\mathrm{P}}\left(\tau+\tau_{\mathrm{d}}\right)^{2}}+\frac{1}{\tau_{\mathrm{d}}\left(k+k_{\mathrm{P}}\right)}\right] \\
& +\frac{6 \tau^{5}}{\tau_{\mathrm{d}}^{2}\left(\tau+\tau_{\mathrm{d}}\right)^{3}} \ln \left[k\left(\tau+\tau_{\mathrm{d}}\right)+k_{\mathrm{P}} \tau\right] \\
& -\frac{6 \tau\left(6 \tau^{2}+8 \tau \tau_{\mathrm{d}}+3 \tau_{\mathrm{d}}^{2}\right)}{\left(\tau+\tau_{\mathrm{d}}\right)^{3}} \ln \left[k_{\mathrm{P}}\right] \\
& \left.-\frac{6 \tau\left(\tau-3 \tau_{\mathrm{d}}\right)}{\tau_{\mathrm{d}}^{2}} \ln \left[k+k_{\mathrm{P}}\right]\right\}+O(1 / \varepsilon) .
\end{aligned}
$$

As for $E_{\mathrm{H}}$, it is defined up to constant. The contribution $E_{\mathrm{Q} 1}$ of the quartic term in the quasistatic work is of order $\varepsilon^{n-2}$ to leading order in $\varepsilon$. Given that this contribution should be negligible with respect to $W_{\mathrm{H}}$, we deduce that $n$ should fulfill the condition $n>1$. The energy scale $E_{\mathrm{Q} 1}$ is affected by the non-Gaussian statistics of the active noise. Given that the tracers' dynamics is nonlinear in $x$, the second moment of the tracers' statistics now depends on higher moments of the active noise which reveal the non-Gaussian nature of the dynamics.

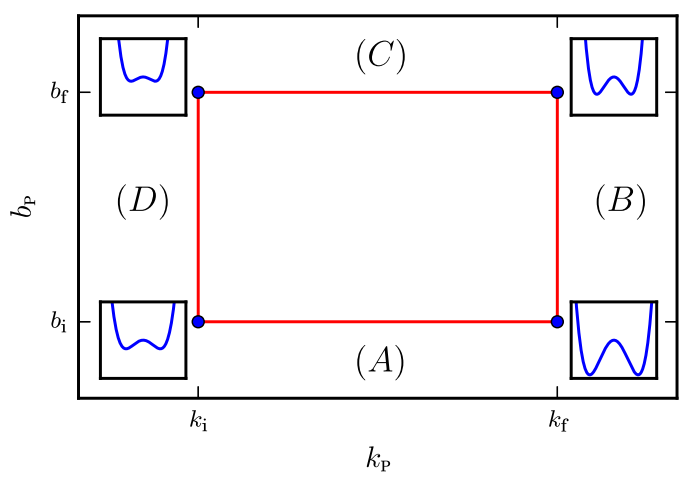

FIG. 5. (Color online) Schematic representation of cycle $\mathcal{C}$. The optical trap parameters undergo the transformations $(A)$ to $(D)$ : $\left\{b_{\mathrm{i}}, k_{\mathrm{i}}\right\} \stackrel{(A)}{\longrightarrow}\left\{b_{\mathrm{i}}, k_{\mathrm{f}}\right\} \stackrel{(B)}{\longrightarrow}\left\{b_{\mathrm{f}}, k_{\mathrm{f}}\right\} \stackrel{(C)}{\longrightarrow}\left\{\beta_{\mathrm{f}}, k_{\mathrm{i}}\right\} \stackrel{(D)}{\longrightarrow}\left\{b_{\mathrm{i}}, k_{\mathrm{i}}\right\}$. The shape of the external potential tuned by the operator is depicted as a function of the position for the four parameter sets, where $k_{\mathrm{f}}=2 k_{\mathrm{i}}<0$ and $b_{\mathrm{f}}=2 b_{\mathrm{i}}$.

We consider a circular protocol $\mathcal{C}$ where both $k_{\mathrm{P}}$ and $b_{\mathrm{P}}$ are modified in time. The simplest protocol is then given by four elementary transformations during which a single parameter is varied, the other one remaining constant. The cycle is illustrated in Fig. 5. It connects four points in the $\left\{k_{\mathrm{P}}, b_{\mathrm{P}}\right\}$ plane:

$$
\left\{b_{\mathrm{i}}, k_{\mathrm{i}}\right\} \stackrel{(A)}{\rightarrow}\left\{b_{\mathrm{i}}, k_{\mathrm{f}}\right\} \stackrel{(B)}{\rightarrow}\left\{b_{\mathrm{f}}, k_{\mathrm{f}}\right\} \stackrel{(C)}{\rightarrow}\left\{b_{\mathrm{f}}, k_{\mathrm{i}}\right\} \stackrel{(D)}{\rightarrow}\left\{b_{\mathrm{i}}, k_{\mathrm{i}}\right\} .
$$

The associated average quasistatic work is defined as

$$
W_{\mathcal{C}}=\frac{1}{2} \oint_{\mathcal{C}} d k_{\mathrm{P}}\left\langle x^{2}\right\rangle_{\mathrm{SS}}+\frac{1}{4} \oint_{\mathcal{C}} d b_{\mathrm{P}}\left\langle x^{4}\right\rangle_{\mathrm{SS}} .
$$

To leading order in $b_{\mathrm{P}}$, the steady-state average $\left\langle x^{4}\right\rangle_{\mathrm{SS}}$ in the above formula is evaluated for a quadratic optical trap, as we compute it in Appendix B 2. It follows the quasistatic work associated with the protocol $\mathcal{C}$ is expressed to leading order in $b_{\mathrm{i}}$ and $b_{\mathrm{f}}$ as

$$
\begin{aligned}
W_{\mathcal{C}}= & E_{\mathrm{Q} 1}\left(k_{\mathrm{f}}, b_{\mathrm{i}}\right)-E_{\mathrm{Q} 1}\left(k_{\mathrm{i}}, b_{\mathrm{i}}\right)+E_{\mathrm{Q} 2}\left(k_{\mathrm{f}}, b_{\mathrm{f}}\right)-E_{\mathrm{Q} 2}\left(k_{\mathrm{f}}, b_{\mathrm{i}}\right) \\
& +E_{\mathrm{Q} 1}\left(k_{\mathrm{i}}, b_{\mathrm{f}}\right)-E_{\mathrm{Q} 1}\left(k_{\mathrm{f}}, b_{\mathrm{f}}\right) \\
& +E_{\mathrm{Q} 2}\left(k_{\mathrm{i}}, b_{\mathrm{i}}\right)-E_{\mathrm{Q} 2}\left(k_{\mathrm{i}}, b_{\mathrm{f}}\right)
\end{aligned}
$$

where $E_{\mathrm{Q} 2}$ is linear in $b_{\mathrm{P}}$ :

$$
\frac{E_{\mathrm{Q} 2}\left(k_{\mathrm{P}}, b_{\mathrm{P}}\right)}{b_{\mathrm{P}}}=3\left[\frac{k T_{\mathrm{A}}}{2 k_{\mathrm{P}}\left(k+k_{\mathrm{P}}\right) \varepsilon}\right]^{2}+O(1 / \varepsilon) .
$$

The formula (13) reveals that one can measure some work for a circular protocol if the external potential applied on the tracer contains an anharmonic component [17]. The equilibrium counterpart of this work vanishes, namely, for the itinerant oscillator case when $T_{\mathrm{A}}=0$, and a nonzero work can thus be regarded as a signature of nonequilibrium activity within the system. The work applied during such a protocol is of order $\varepsilon^{n-2}$ to leading order in $\varepsilon$. Being $n$ necessarily greater than 1 , we deduce this work is negligible compared with the work associated to the protocol presented in Sec. III. Thus, the anharmonicity of the external potential leads to a nonzero quasistatic work for a circular protocol, but its small value may be hard to measure experimentally. 
Assuming an experimental apparatus can actually detect such a work, the active temperature can then be extracted from this measurement, given that the backaction strength $\varepsilon$ has been estimated by another method and the parameter $n$ is controlled by the operator. A simple method to fix $n$ is to tune the anharmonicity so that it gives a nonzero contribution to $W_{\mathrm{Q}}-T_{\mathrm{A}} \ln \left[k_{\mathrm{f}}\left(k+k_{\mathrm{i}}\right) / k_{\mathrm{i}} /\left(k+k_{\mathrm{f}}\right)\right] /(2 \varepsilon)$ by detecting when the value of this work differs from the order $\varepsilon^{0}$ in $W_{\mathrm{H}}$. In such a case, the contribution of $E_{\mathrm{Q} 1}$ is to be taken into account, so that it corresponds to the case $n=2$. Note that neither $E_{\mathrm{Q} 1}$ nor $E_{\mathrm{Q} 2}$ depends on $\tau_{0}$ to leading order in $\varepsilon$. Then, the expressions we present would remain unchanged in the limit $\tau_{0} \rightarrow 0$, where the active noise is a symmetric two-state process usually referred to as a random telegraphic noise [34]. The waiting time scale $\tau_{0}$ affects the next order in $\varepsilon$ of the work associated with the cycle $\mathcal{C}$. Moreover, the work applied during such a protocol does not vanish in the limit where the active process $v_{\mathrm{A}}$ becomes a white noise, namely when $\left\{\tau, \tau_{0}, v\right\} \rightarrow\{0,0, \infty\}$ with fixed $T_{\mathrm{A}}$. In such a limit and assuming $T=0$, the dynamics presented in Eq. (3) describes the evolution of a particle subjected to a white non-Gaussian noise, so that one can indeed extract work from a cycle as already noticed in [17].

\section{EFFECTIVE TEMPERATURE AND FORCE-POSITION CORRELATIONS}

Active microrheology experiments on living cells measure the response $\chi$ to an external stress, and its temporal Fourier transform $\tilde{\chi}(\omega)=\int d t e^{-i \omega t} \chi(t)$. The latter is, up to a constant, the inverse of the complex modulus $G^{*}[35]: G^{*}=1 /[6 \pi a \tilde{\chi}]$, where $\tilde{\chi}$ is the response function in the Fourier domain. We assume that motor activity does not affect the rheological properties of the network, so that we deduce the response function from the expression of the complex modulus in a passive system as presented in Eq. (2). Following Lau et al. [36], the tracer's evolution in a viscous fluid is modeled as

$$
\gamma \frac{d x}{d t}=F_{\text {cell }}(t)
$$

where $F_{\text {cell }}$ describes all the forces arising from the medium. Within this minimal assumption several works have measured the nonequilibrium properties of the force $F_{\text {cell }}[11,16]$. These were quantified by looking at the deviation from such equilibrium relations as the FDT. For example, the correlationto-response ratio leads to a frequency-dependent "effective temperature" as [12-14] $T_{\mathrm{eff}}(\omega)=-\omega \tilde{C}(\omega) /\left[2 \tilde{\chi}^{\prime \prime}(\omega)\right]$, where $\tilde{\chi}^{\prime \prime}$ is the imaginary part of the response Fourier transform and $\tilde{C}$ is the position autocorrelation function in the Fourier domain. Of course, this effective temperature is not a bona fide temperature, in the sense that even in a stationary regime it is generally observable-dependent, but the fact that its high frequency value collapses to the bath temperature in the absence of nonequilibrium processes constitutes a useful benchmark. This is the simplest manner to evaluate the distance from equilibrium. In the absence of external potential as described in Eq. (3), we compute analytically this temperature to leading order in $\varepsilon$ :

$$
T_{\text {eff }}(\omega)=T+\frac{1}{\varepsilon+\left(\omega \tau_{\mathrm{d}}\right)^{2}} \frac{T_{\mathrm{A}}}{1+(\omega \tau)^{2}} .
$$
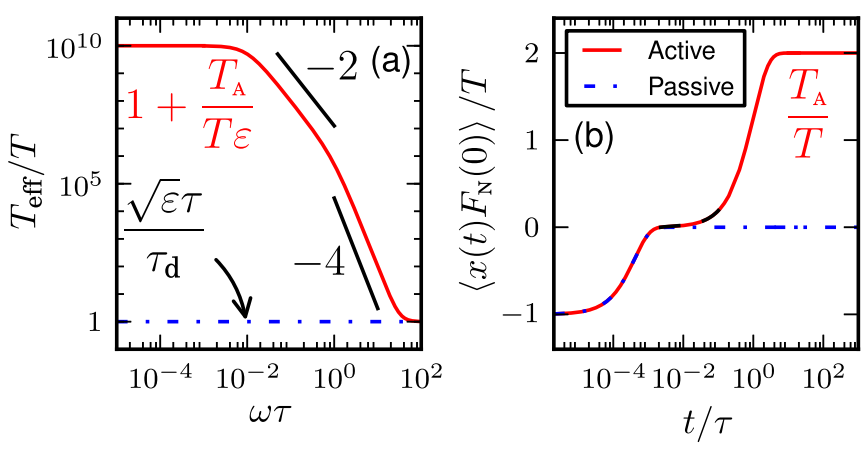

FIG. 6. (Color online) (a) Effective temperature as a function of the scaled frequency $\omega \tau$. The plateau value at low frequency equals $T+T_{\mathrm{A}} / \varepsilon$ (red solid line), and it equals $T$ at high frequency as for the passive case (blue dot-dashed line). Between the two saturations, it scales successively like $1 / \omega^{2}$ and $1 / \omega^{4}$, provided the time scales $\tau$ and $\tau_{\mathrm{d}} / \sqrt{\varepsilon}$ are well separated. (b) Evolution of the force-position correlation function with the scaled time $t / \tau$ in the passive (blue dotdashed line) and active (red solid line) cases. The correlation function is negative at short time scale with an initial value $-T$. It remains negative in the passive case. There is a linear growth regime in the active case (black dashed line), and the correlation function saturates to a plateau value $T_{\mathrm{A}}$. (a) $\left\{T, \varepsilon, \tau_{\mathrm{d}}, T_{\mathrm{A}}, \tau\right\}=\left\{1,10^{-8}, 1,10^{2}, 10^{2}\right\}$. (b) $\left\{T, \tau_{\mathrm{d}}, T_{\mathrm{A}}, \tau\right\}=\left\{5,0.2,10,5 \times 10^{2}\right\}$.

At high frequencies, the effective temperature coincides with the bath temperature $T$, meaning thermal fluctuations are predominant with respect to motor activity in this regime, in agreement with the MSD short time behavior. The plateau value $T+T_{\mathrm{A}} / \varepsilon$ at low frequency represents an alternative measurement of the active fluctuation amplitude. Between the two plateaus, the effective temperature successively scales like $1 / \omega^{4}$ and $1 / \omega^{2}$ given that the two time scales $\tau$ and $\tau_{\mathrm{d}} / \sqrt{\varepsilon}$ are well separated as shown in Fig. 6(a), thus providing a way to determine these time scale values from the slope variation. When we neglect the backaction effect, the effective temperature diverges at low frequencies. It results from the fact that the active MSD diffuses at large time scale, whereas it saturates to the equilibrium value for a passive system. The introduction of the backaction changes the rheology of the material, so that the passive MSD also diffuses at a large time scale, from which we deduce the effective temperature saturates at low frequency.

A generalization of usual microrheology measurements relies on applying an arbitrary perturbation on the tracers and measuring their response function. The external stimulus is generally a homogeneous force. We address here the case where an arbitrary potential $V_{\mathrm{P}}=-a_{\mathrm{P}}(t) V(x(t))$ is applied on the tracers. The generalized tracers' response $\chi_{\mathrm{G}}$ quantifies the effect of the perturbation on an arbitrary observable $A$ :

$$
\chi_{\mathrm{G}}(s, u)=\left.\frac{\delta\langle A(s)\rangle}{\delta a_{\mathrm{P}}(u)}\right|_{a_{\mathrm{P}}=0} .
$$

Causality ensures that the response function is zero when the measurement is performed before the perturbation, at $u<s$. Since the thermal noise has Gaussian statistics, the probability weight $\mathcal{P}$ associated with a given realization of the thermal noise is defined as $\mathcal{P}[\xi] \propto e^{-\mathcal{S}[\xi]}$, where $\mathcal{S}[\xi]=\int d t^{\prime} \xi^{2}\left(t^{\prime}\right) / 2$ is the Onsager-Machlup (or action) functional, in which $\xi$ 
determines the dynamics of the probe $[18,19]$. The application of the external potential $V_{\mathrm{P}}$ results in a variation $\delta \mathcal{S}$ of the action functional, so that the response function is expressed as

$$
\chi_{\mathrm{G}}(s, u)=-\left\langle\left. A(s) \frac{\delta \mathcal{S}}{\delta a_{\mathrm{P}}(u)}\right|_{a_{\mathrm{P}}=0}\right\rangle .
$$

To determine the response function, we only need to compute the action functional to leading order in $a_{\mathrm{P}}$,

$$
\mathcal{S}=-\int d t^{\prime} \frac{a_{\mathrm{P}}\left(t^{\prime}\right)}{2 \gamma T}\left[\gamma \frac{d x}{d t^{\prime}}-F_{\mathrm{N}}\left(t^{\prime}\right)\right] \frac{d V\left(x\left(t^{\prime}\right)\right)}{d x}+O\left(a_{\mathrm{P}}^{2}\right),
$$

where $F_{\mathrm{N}}=-k\left(x-x_{0}\right)$ is the force reflecting the interaction of the tracer with the surrounding actin network. We deduce the response function in terms of the probe's statistics and the network force,

$$
\chi_{\mathrm{G}}(s, u)=\frac{1}{2 \gamma T}\left[\gamma \frac{\partial C_{\mathrm{AV}}(s, u)}{\partial u}-\left\langle A(s) \frac{d V(x(u))}{d x} F_{\mathrm{N}}(u)\right\rangle\right],
$$

where $C_{\mathrm{AV}}(s, u)=\langle A(s) V(u)\rangle$. This expression reveals that one can gain information about the correlation between the network force and the tracers' statistics by independently measuring $\chi_{\mathrm{G}}$ and $C_{\mathrm{AV}}$.

In the case where $a_{\mathrm{P}}$ is a homogeneous force, when $V_{\mathrm{P}}=-a_{\mathrm{P}} x$, the response function is measured by usual microrheology methods. If we choose the observable $A$ to be the tracers' position $x$, it is possible to access the force-position correlation $\left\langle x(s) F_{\mathrm{N}}(u)\right\rangle$ [18]. After an exponentially fast initial transient regime which we neglect, this correlation function depends only on the lag time $t=s-u$. The expression for this correlation is not invariant under time reversal, and we compute it for the case $t>0$ to leading order in $\varepsilon$ :

$$
\begin{aligned}
\left\langle x(t) F_{\mathrm{N}}(0)\right\rangle= & -T e^{-t / \tau_{\mathrm{d}}}+\frac{T_{\mathrm{A}}}{1-\left(\tau / \tau_{\mathrm{d}}\right)^{2}} \\
& \times\left[1-e^{-t / \tau_{\mathrm{d}}}-\left(\frac{\tau}{\tau_{\mathrm{d}}}\right)^{2}\left(1-e^{-t / \tau}\right)\right] .
\end{aligned}
$$

The initial value $-T$ is negative and equals the thermal fluctuation amplitude in agreement with [18]. This anticorrelation between the network force and the tracers' displacement is another evidence of the short time scale confinement. In the active case, the correlation function can take positive values, showing that the active burst allows the tracer to overcome the short time scale confinement. When $\tau \gg \tau_{\mathrm{d}}$, there is a linear growth with coefficient $T_{\mathrm{A}} / \tau /\left[1-\left(\tau_{\mathrm{d}} / \tau\right)^{2}\right]$, and then it reaches a plateau value $T_{\mathrm{A}}$ as presented in Fig. 6(b). The linear regime is observed in [18], but the plateau is not present. We speculate that a larger time window would allow one to observe the saturation of the correlation function. The existence of the plateau calls for new experiments as it would provide yet another way of measuring the amplitude of active fluctuations. Note that this amplitude is also accessible via the linear growth coefficient if $\tau$ and $\tau_{\mathrm{d}}$ are already known. Moreover, a positive value of the force-position correlation function is a signature of nonequilibrium activity within the system as it would remain negative for an equilibrium process.

\section{ENERGY DISSIPATION AND HARADA-SASA RELATIONS}

The dissipation within the system is the work applied by the tracer on the surrounding environment regarded as a heat bath [33]. It has already been measured in colloidal systems $[37,38]$ and should be a good criterion to characterize nonequilibrium activity in biological systems. We adopt a natural definition for the mean rate of energy dissipation [32,33]: $J=\langle\dot{x}(\gamma \dot{x}-\xi)\rangle$, where $\dot{x}$ is the velocity of the tracer. It is the difference between the mean power given by the particle to the heat bath via the drag force $\gamma \dot{x}$ and the one provided in average by the thermostat to the particle via the thermal force $\xi$. It has been demonstrated by Harada and Sasa that this quantity is related to the correlation and response functions defined previously [20]: $J=\gamma \int d \omega\left[\omega \tilde{C}(\omega)+2 T \tilde{\chi}^{\prime \prime}(\omega)\right] \omega /(2 \pi)$. This relation presents the heat current $J$ as a quantification of the deviation from the FDT valid for an equilibrium process. Within our model, the energy dissipation rate equals the average power of the network force: $J=\left\langle\dot{x} F_{\mathrm{N}}\right\rangle$. We compute it in terms of the microscopic ingredients:

$$
J=\frac{T_{\mathrm{A}}}{\tau+\tau_{\mathrm{d}}} .
$$

It is not affected by the backaction to leading order in $\varepsilon$. The energy dissipation rate is zero when no activity occurs in the medium for an arbitrary value of $\varepsilon$, as expected for an equilibrium process. The dissipation rate depends on the coupling between the probe and its environment via $\tau_{\mathrm{d}}$. To minimize the dissipation rate, the time scale of the quiescent periods $\tau_{0}$ should be as large as possible, whereas the time scale of the ballistic jumps $\tau$ should be very small, in agreement with observations in biological systems for which $\tau_{0}>\tau[3,11]$. As in the previous section, the definition and the expression of $J$ show that one can access the microscopic features of motor activity via independent measurements of the correlation and response functions.

The main drawback of this approach is that one should measure $\tilde{C}$ and $\tilde{\chi}$ over a large range of frequencies to access the energy dissipation rate. Thus, it is interesting to focus on the spectral density of the energy dissipation rate, $\tilde{I}(\omega)=\gamma \omega\left[\omega \tilde{C}(\omega)+2 T \tilde{\chi}^{\prime \prime}(\omega)\right]$, which, when integrated over the whole frequency range, equals the energy dissipation rate [20,37]: $J=\int d \omega \tilde{I}(\omega) /(2 \pi)$. To give a physical interpretation of this quantity, we introduce the operators $\theta_{ \pm}$the effect of which on an arbitrary function $f(t)$ is to extract its even/odd component: $\theta_{ \pm}[f(t)]=[f(t) \pm f(-t)] / 2$. The Fourier transform of the symmetrized force-velocity correlation function is $\tilde{I}[20,39]$, so that $I(t)=\theta_{+}\left[\left\langle\dot{x}(t) F_{\mathrm{N}}(0)\right\rangle\right]$. This relation is a reformulation of Eq. (21) when $V=x=A$, and we see in which sense it enables one to easily access the characteristics of motor activity. Note that the antisymmetrized force-position correlation function defined previously is also related to this quantity: $I(t)=d \theta_{-}\left[\left\langle x(t) F_{\mathrm{N}}(0)\right\rangle\right] / d t$. We compute the dissipation rate spectrum analytically to leading order in $\varepsilon$ :

$$
\tilde{I}(\omega)=\frac{1}{1+\left(\omega \tau_{\mathrm{d}}\right)^{2}} \frac{2 T_{\mathrm{A}}}{1+(\omega \tau)^{2}} .
$$

The low frequency plateau provides a direct measurement of the active fluctuation amplitude $T_{\mathrm{A}}$. At high frequency, it scales 

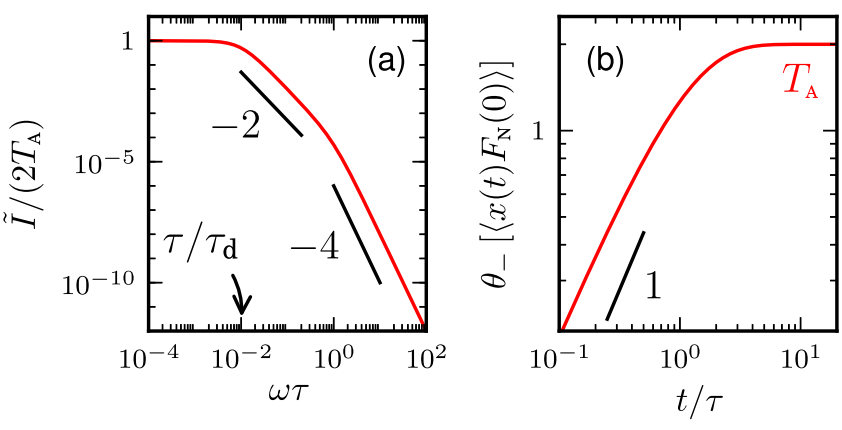

FIG. 7. (Color online) (a) Evolution of the Fourier transform of the spectral density of the energy dissipation rate with the scaled frequency $\omega \tau$ when $\tau \gg \tau_{\mathrm{d}}$. The plateau value at low frequency equals $2 T_{\mathrm{A}}$. It scales like $1 / \omega^{4}$ at high frequency, and there is a crossover regime $1 / \tau_{\mathrm{d}} \ll \omega \ll 1 / \tau$ with another power law $1 / \omega^{2}$. (b) Antisymmetric force-position correlation function as a function of the scaled time $t / \tau$. It is linear in time at short time scale with a growth coefficient $J$ and saturates to the value $T_{\mathrm{A}}$ at large time scale. $\left\{T_{\mathrm{A}}, \tau, \tau_{\mathrm{d}}\right\}=\{2,10,0.1\}$.

like $1 / \omega^{4}$, and there is a crossover regime $\omega_{1} \ll \omega \ll \omega_{2}$, where $\omega_{1}, \omega_{2} \in\left\{1 / \tau, 1 / \tau_{\mathrm{d}}\right\}$, with a power law behavior $1 / \omega^{2}$. Thus, one can determine $\tau$ and $\tau_{\mathrm{d}}$ from the variation of the slope, provided the two time scales are well separated, as presented in Fig. 7(a). We derive the antisymmetric forceposition correlation function from $\tilde{I}$ to leading order in $\varepsilon$ :

$$
\begin{aligned}
\theta_{-} & {\left[\left\langle x(t) F_{\mathrm{N}}(0)\right\rangle\right] } \\
= & \frac{T_{\mathrm{A}}}{1-\left(\tau / \tau_{\mathrm{d}}\right)^{2}}\left[1-e^{-t / \tau_{\mathrm{d}}}-\left(\frac{\tau}{\tau_{\mathrm{d}}}\right)^{2}\left(1-e^{-t / \tau}\right)\right] .
\end{aligned}
$$

It equals the force-position correlation function in Eq. (21) when $T=0$. At short time scale, growth is linear with a coefficient $J$. Hence, it is possible to estimate the energy dissipation rate by measuring its spectral density only in the high frequency domain, which mostly facilitates the experimental task with respect to the procedure proposed in [20]. The correlation function saturates to a plateau value $T_{\mathrm{A}}$ at large time scale as presented in Fig. 7(b), showing that it provides an alternative to directly measuring both the energy dissipation rate and the amplitude of the tracer's active fluctuations.

\section{CONCLUSION}

We offer theoretical predictions for energetic observables of a system where both thermal fluctuations and nonequilibrium activity coexist. We also propose a set of concrete experimental methods and protocols so that our predictions may be tested with existing experimental techniques. These new methods end up in more stringent constraints on the theoretical modeling which is employed in the studies of tracer dynamics, and thus they should also be a crucial test for the robustness of our own model. By applying such methods, we find that one can access the microscopic features of motor activity and fully characterize the nonequilibrium process arising in the medium. The most natural step forward is to address the analytic computation of the finite time extracted work, for which one should find an optimal protocol maximizing the extracted power [40]. Another interesting issue is the excess heat and housekeeping heat produced by such a protocol, the computation of which requires the determination of the steadystate distribution of the process [41]. The bath temperature could be regarded as another tunable parameter provided its variation does not modify the microscopic features of the system [42], which is not the case in biological systems but could be conceivable in colloidal systems. Finally, the rheology of living matter can be more complex than we propose in this paper [43,44]. Memory effects arise from the interaction of the particle with the environment, due to the integration of some additional degrees of freedom, leading to a power law behavior for the complex modulus. Dressing the tracers' dynamics with a more realistic rheology should be included in a future elaboration of the model.

\section{ACKNOWLEDGMENTS}

Part of the numerical calculations was carried out on SR16000 at YITP in Kyoto University. This work was supported by the JSPS Core-to-Core Program Non-equilibrium Dynamics of Soft Matter and Information, the Grants-inAid for Japan Society for Promotion of Science (JSPS) Fellows (Grant No. 24.3751), and JSPS KAKENHI Grant No. 22340114.

\section{APPENDIX A: ACTIVE BURST STATISTICS}

We denote $P_{\text {off }}$ the transition probability to the state in which $v_{\mathrm{A}}$ is zero, and $P_{\mathrm{on}}$ the transition probability to the state $v_{\mathrm{A}}=p v$, where $p$ is a uniform random value between -1 and 1 . The set of master equations describing the evolution of the active burst one dimensional projection is

$$
\begin{aligned}
d_{t} P_{\mathrm{off}}(t) & =\frac{1}{\tau}-P_{\mathrm{off}}(t)\left(\frac{1}{\tau}+\frac{1}{\tau_{0}}\right), \\
\partial_{t} P_{\mathrm{on}}(t, p) & =\frac{P_{\mathrm{off}}(t)}{2 \tau_{0}}-\frac{P_{\mathrm{on}}(t, p)}{\tau} .
\end{aligned}
$$

We derive the expression of the transition probability $P_{\text {on }}$ from these equations. For symmetry reasons, only the $2 n$-time correlation functions of the active burst are nonzero. Given that the active burst is in the steady state at the initial time, the $2 n$-time correlation function $K_{\mathrm{A}}\left(\left\{t_{i}\right\}\right)=\left\langle v_{\mathrm{A}}\left(t_{2 n}\right) v_{\mathrm{A}}\left(t_{2 n-1}\right) \ldots v_{\mathrm{A}}\left(t_{1}\right)\right\rangle$ reads

$$
\frac{K_{\mathrm{A}}\left(\left\{t_{i}\right\}\right)}{v^{2 n}}=\int d^{2 n} p P_{\mathrm{on}}^{\mathrm{ss}}\left(p_{1}\right) p_{1} \prod_{i=2}^{2 n} P_{\mathrm{on}}\left(t_{i}-t_{i-1}, p_{i} \mid p_{i-1}\right) p_{i},
$$

where $P_{\mathrm{on}}\left(t, p_{b} \mid p_{a}\right)$ is the transition probability from $p_{a}$ to $p_{b}$, and $P_{\mathrm{on}}^{\mathrm{ss}}$ is the steady-state transition probability. We deduce the explicit expression of $K_{\mathrm{A}}$,

$$
K_{\mathrm{A}}\left(\left\{t_{i}\right\}\right)=\phi\left(t_{2}-t_{1}\right) \prod_{i=1}^{n-2} \phi\left(t_{2 i+2}-t_{2 i+1}\right) \psi\left(t_{2 i+1}-t_{2 i}\right),
$$


where $t_{2 n} \geqslant t_{2 n-1} \geqslant \cdots \geqslant t_{1}$. The functions $\phi$ and $\psi$ are defined as

$$
\begin{aligned}
\phi(t) & =\frac{v^{2} p_{\text {on }}}{3} e^{-|t| / \tau} \\
\psi(t) & =1+\frac{4}{5}\left(1+\frac{\tau_{0}}{\tau}\right) e^{-|t| / \tau}+\frac{\tau_{0}}{\tau} e^{-|t|\left(1 / \tau+1 / \tau_{0}\right)} .
\end{aligned}
$$

\section{APPENDIX B: TRACER STATISTICS}

\section{Without optical trap}

We compute the expression of the MSD for the dynamics without optical trap in Eq. (3) for an arbitrary value of $\varepsilon$,

$$
\begin{aligned}
\left\langle\Delta x_{\mathrm{T}}^{2}\right\rangle(t)= & \frac{2 T / k}{(1+\varepsilon)^{2}}\left(1-e^{-t / \tau_{\varepsilon}}+\varepsilon \frac{t}{\tau_{\varepsilon}}\right), \\
\left\langle\Delta x_{\mathrm{A}}^{2}\right\rangle(t)= & \frac{2 T_{\mathrm{A}} /\left[k(1+\varepsilon)^{3}\right]}{1-\left(\tau / \tau_{\varepsilon}\right)^{2}}\left[e^{-t / \tau_{\varepsilon}}+\frac{t}{\tau_{\varepsilon}}-1\right. \\
& \left.+\left(\frac{\tau}{\tau_{\varepsilon}}\right)^{3}\left(1-e^{-t / \tau}-\frac{t}{\tau}\right)\right],
\end{aligned}
$$

where $\tau_{\varepsilon}=\tau_{\mathrm{d}} /(1+\varepsilon)$.

\section{Quadratic optical trap}

The dynamics of $x$ and $x_{0}$ is given by the set of equations

$$
\begin{aligned}
\frac{d x}{d t} & =-\frac{1}{\tau_{\mathrm{d}}}\left(x-x_{0}\right)-\frac{1}{\tau_{\mathrm{P}}} x+\sqrt{2 D_{\mathrm{T}}} \xi, \\
\frac{d x_{0}}{d t} & =-\frac{\varepsilon}{\tau_{\mathrm{d}}}\left(x_{0}-x\right)+v_{\mathrm{A}}+\sqrt{2 \varepsilon D_{\mathrm{T}}} \xi_{0},
\end{aligned}
$$

where $\tau_{\mathrm{P}}=\gamma / k_{\mathrm{P}}$. Given that we are interested in the regime where the dynamics is time translational invariant, it should not depend on initial condition, so that we can choose $x(0)=0=x_{0}(0)$. Using the Fourier transform of Eq. (B2), we express the tracer's position in terms of the stochastic noises in the Fourier domain as

$$
\tilde{x}=\gamma\left[\tilde{\chi} \sqrt{2 D_{\mathrm{T}}} \tilde{\xi}+\tilde{\chi}_{\mathrm{A}}\left(\tilde{v}_{\mathrm{A}}+\sqrt{2 \varepsilon D_{\mathrm{T}}} \tilde{\xi}_{0}\right)\right],
$$

where the functions $\tilde{\chi}$ and $\tilde{\chi}_{\mathrm{A}}$ are defined as

$$
\begin{aligned}
\tilde{\chi}(\omega) & =\frac{\left(\varepsilon+i \omega \tau_{\mathrm{d}}\right) / k}{i \omega \tau_{\mathrm{d}}\left(1+\varepsilon+i \omega \tau_{\mathrm{d}}\right)+k_{\mathrm{P}}\left(\varepsilon+i \omega \tau_{\mathrm{d}}\right) / k}, \\
\tilde{\chi}_{\mathrm{A}}(\omega) & =\frac{1 / k}{i \omega \tau_{\mathrm{d}}\left(1+\varepsilon+i \omega \tau_{\mathrm{d}}\right)+k_{\mathrm{P}}\left(\varepsilon+i \omega \tau_{\mathrm{d}}\right) / k} .
\end{aligned}
$$

Alternatively, the tracer's position is expressed in the time domain as

$$
\begin{aligned}
x(t)= & \gamma \int^{t} d t^{\prime}\left\{\chi\left(t-t^{\prime}\right) \sqrt{2 D_{\mathrm{T}}} \xi\left(t^{\prime}\right)\right. \\
& \left.+\chi_{\mathrm{A}}\left(t-t^{\prime}\right)\left[v_{\mathrm{A}}\left(t^{\prime}\right)+\sqrt{2 \varepsilon D_{\mathrm{T}}} \xi_{0}\left(t^{\prime}\right)\right]\right\} .
\end{aligned}
$$

By using the residue theorem, we compute from Eq. (B4) the expression of $\chi$ and $\chi_{\mathrm{A}}$ in the time domain,

$$
\begin{aligned}
\chi(t) & =\frac{1}{\gamma\left(c_{+}-c_{-}\right)}\left(c_{+} e^{-t / \tau_{+}}-c_{-} e^{-t / \tau_{-}}\right), \\
\chi_{\mathrm{A}}(t) & =\frac{1}{\gamma\left(c_{+}-c_{-}\right)}\left(e^{-t / \tau_{+}}-e^{-t / \tau_{-}}\right),
\end{aligned}
$$

where $\tau_{ \pm}=\tau_{\mathrm{d}} /\left(\varepsilon-c_{ \pm}\right)$, and the coefficients $c_{ \pm}$read

$$
c_{ \pm}=\frac{\varepsilon-1-k_{\mathrm{P}} / k}{2}\left[1 \pm \sqrt{1+\frac{4 \varepsilon}{\left(\varepsilon-1-k_{\mathrm{P}} / k\right)^{2}}}\right] .
$$

We determine the position autocorrelation function in the Fourier domain for an arbitrary $\varepsilon$ :

$$
\begin{aligned}
\tilde{C}(\omega)= & \frac{2\left(\tau_{+} \tau_{-}\right)^{2} /\left(k \tau_{\mathrm{d}}^{3}\right)}{\left[1+\left(\tau_{+} \omega\right)^{2}\right]\left[1+\left(\tau_{-} \omega\right)^{2}\right]} \\
& \times\left\{\left[\varepsilon+\varepsilon^{2}+\left(\omega \tau_{\mathrm{d}}\right)^{2}\right] T+\frac{T_{\mathrm{A}}}{1+(\omega \tau)^{2}}\right\} .
\end{aligned}
$$

We then deduce the expression of the MSD, without any assumption made on $\varepsilon$,

$$
\begin{aligned}
\left\langle\Delta x_{\mathrm{T}}^{2}\right\rangle(t)= & \frac{2 T / k}{\left(c_{+}-c_{-}\right)\left(c_{-}+c_{+}-2 \varepsilon\right)} \\
& \times\left[\frac{c_{-}^{2}-\varepsilon\left(1+2 c_{-}\right)}{c_{-}-\varepsilon}\left(1-e^{-t / \tau_{-}}\right)\right. \\
& \left.-\frac{c_{+}^{2}-\varepsilon\left(1+2 c_{+}\right)}{c_{+}-\varepsilon}\left(1-e^{-t / \tau_{+}}\right)\right], \\
\left\langle\Delta x_{\mathrm{A}}^{2}\right\rangle(t)= & \frac{2 T_{\mathrm{A}} / k}{\left[\left(\tau / \tau_{-}\right)^{2}-1\right]\left[\left(\tau / \tau_{+}\right)^{2}-1\right]\left(c_{+}-c_{-}\right)} \\
& \times\left[\left(c_{+}-c_{-}\right)\left(1-e^{-t / \tau}\right)\left(\frac{\tau}{\tau_{\mathrm{d}}}\right)^{3}\right. \\
& +\frac{\left(\tau / \tau_{+}\right)^{2}-1}{c_{+}+c_{-}-2 \varepsilon}\left(1-e^{-t / \tau_{-}}\right) \\
& \left.-\frac{\left(\tau / \tau_{-}\right)^{2}-1}{c_{-}+c_{+}-2 \varepsilon}\left(1-e^{-t / \tau_{+}}\right)\right] .
\end{aligned}
$$

From the saturation value of the MSD at large time scale, we deduce the expression of the steady-state average:

$$
\begin{aligned}
\left\langle x^{2}\right\rangle_{\mathrm{SS}}= & \frac{T_{\mathrm{A}} k}{\varepsilon k_{\mathrm{P}}\left(k+k_{\mathrm{P}}\right)} \\
& +\frac{T}{k_{\mathrm{P}}}-\frac{T_{\mathrm{A}} k}{\left(k+k_{\mathrm{P}}\right)^{2}}\left[\frac{\tau}{\tau_{\mathrm{d}}}+\frac{k^{2}\left(\tau+\tau_{\mathrm{d}}\right)}{k_{\mathrm{P}}\left(k+k_{\mathrm{P}}\right) \tau+k k_{\mathrm{P}} \tau_{\mathrm{d}}}\right] \\
& +O(\varepsilon) .
\end{aligned}
$$

The expression of $E_{\mathrm{H}}$ is given by the primitive of the above formula with respect to $k_{\mathrm{P}}$, thus being defined up to a constant. To determine the non-Gaussian parameter, we compute the

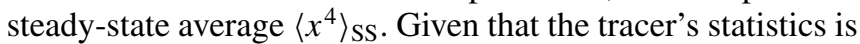
Gaussian to leading order in $\varepsilon$, we can easily deduce $\left\langle x^{4}\right\rangle_{\text {SS }}$ to first order in $\varepsilon$ from the above formula:

$$
\left\langle x^{4}\right\rangle_{\mathrm{SS}}=3\left[\frac{k T_{\mathrm{A}}}{\varepsilon k_{\mathrm{P}}\left(k+k_{\mathrm{P}}\right)}\right]^{2}+O(1 / \varepsilon) .
$$


The computation of the next order requires to develop the expression of $x^{4}$ in terms of $\chi$ and $\chi_{\mathrm{A}}$. From Eq. (B5), we split the steady-state average in two contributions:

$$
\left\langle x^{4}\right\rangle_{\mathrm{SS}}=\lim _{t \rightarrow \infty}\left(\kappa_{1}+6 \kappa_{2}\right)(t)
$$

The functions $\kappa_{1}$ and $\kappa_{2}$ read

$$
\begin{aligned}
\kappa_{1}(u)= & \int^{u} \iiint d u_{1} d u_{2} d u_{3} d u_{4}\left[\chi_{\mathrm{A} 1} \chi_{\mathrm{A} 2} \chi_{\mathrm{A} 3} \chi_{\mathrm{A} 4}\left\langle v_{\mathrm{A}}\left(u_{1}\right) v_{\mathrm{A}}\left(u_{2}\right) v_{\mathrm{A}}\left(u_{3}\right) v_{\mathrm{A}}\left(u_{4}\right)\right\rangle\right. \\
& \left.+4\left(\varepsilon D_{\mathrm{T}}\right)^{2} \chi_{\mathrm{A} 1} \chi_{\mathrm{A} 2} \chi_{\mathrm{A} 3} \chi_{\mathrm{A} 4}\left\langle\xi_{0}\left(u_{1}\right) \xi_{0}\left(u_{2}\right) \xi_{0}\left(u_{3}\right) \xi_{0}\left(u_{4}\right)\right\rangle+4 D_{\mathrm{T}}^{2} \chi_{1} \chi_{2} \chi_{3} \chi_{4}\left\langle\xi\left(u_{1}\right) \xi\left(u_{2}\right) \xi\left(u_{3}\right) \xi\left(u_{4}\right)\right\rangle\right] \\
\kappa_{2}(u)= & \int^{u} \iiint d u_{1} d u_{2} d u_{3} d u_{4}\left[2 \varepsilon D_{\mathrm{T}} \chi_{\mathrm{A} 1} \chi_{\mathrm{A} 2} \chi_{\mathrm{A} 3} \chi_{\mathrm{A} 4}\left\langle v_{\mathrm{A}}\left(u_{1}\right) v_{\mathrm{A}}\left(u_{2}\right)\right\rangle\left\langle\xi_{0}\left(u_{3}\right) \xi_{0}\left(u_{4}\right)\right\rangle\right. \\
& \left.+2 D_{\mathrm{T}} \chi_{\mathrm{A} 1} \chi_{\mathrm{A} 2} \chi_{3} \chi_{4}\left\langle v_{\mathrm{A}}\left(u_{1}\right) v_{\mathrm{A}}\left(u_{2}\right)\right\rangle\left\langle\xi\left(u_{3}\right) \xi\left(u_{4}\right)\right\rangle+4 \varepsilon D_{\mathrm{T}}^{2} \chi_{\mathrm{A} 1} \chi_{\mathrm{A} 2} \chi_{3} \chi_{4}\left\langle\xi_{0}\left(u_{1}\right) \xi_{0}\left(u_{2}\right)\right\rangle\left\langle\xi\left(u_{3}\right) \xi\left(u_{4}\right)\right\rangle\right]
\end{aligned}
$$

where $\chi_{i}=\chi\left(u-u_{i}\right), \chi_{\mathrm{A} i}=\chi_{\mathrm{A}}\left(u-u_{i}\right)$, and $i \in\{1,2,3,4\}$. The non-Gaussianity of the active bursts plays a role in the first term in the bracket of Eq. (B13a). Being $\xi$ and $\xi_{0}$ thermal noises, their four-time correlation function is expressed in terms of their two-time correlation function as

$$
\begin{aligned}
\left\langle\xi\left(t_{\mathrm{a}}\right) \xi\left(t_{\mathrm{b}}\right) \xi\left(t_{\mathrm{c}}\right) \xi\left(t_{\mathrm{d}}\right)\right\rangle= & \left\langle\xi\left(t_{\mathrm{a}}\right) \xi\left(t_{\mathrm{b}}\right)\right\rangle\left\langle\xi\left(t_{\mathrm{c}}\right) \xi\left(t_{\mathrm{d}}\right)\right\rangle \\
& +\left\langle\xi\left(t_{\mathrm{a}}\right) \xi\left(t_{\mathrm{c}}\right)\right\rangle\left\langle\xi\left(t_{\mathrm{d}}\right) \xi\left(t_{\mathrm{b}}\right)\right\rangle \\
& +\left\langle\xi\left(t_{\mathrm{a}}\right) \xi\left(t_{\mathrm{d}}\right)\right\rangle\left\langle\xi\left(t_{\mathrm{c}}\right) \xi\left(t_{\mathrm{b}}\right)\right\rangle,
\end{aligned}
$$

and the same property holds for the correlations of $\xi_{0}$. By using Eqs. (A3) and (B14), we finally deduce the next orders in the expression of $\left\langle x^{4}\right\rangle_{\text {SS }}$.

\section{Quartic optical trap}

To compute the steady-state average $\left\langle x^{2}\right\rangle_{\mathrm{SS}}$, we expand the positions $x$ and $x_{0}$ in terms of $b_{\mathrm{P}}$ as $x=x^{(0)}+x^{(1)}+O\left(b_{\mathrm{P}}^{2}\right)$ and $x_{0}=x_{0}^{(0)}+x_{0}^{(1)}+O\left(b_{\mathrm{P}}^{2}\right)$, where $x^{(1)}$ and $x_{0}^{(1)}$ are of order $b_{\mathrm{P}}$. The steady-state average is expressed as

$$
\left\langle x^{2}\right\rangle_{\mathrm{SS}}=\left\langle\left(x^{(0)}\right)^{2}\right\rangle_{\mathrm{SS}}+2\left\langle x^{(0)} x^{(1)}\right\rangle_{\mathrm{SS}}+O\left(b_{\mathrm{P}}^{2}\right) \text {. }
$$

The leading order in $b_{\mathrm{P}}$ equals the steady-state average without quartic term in the optical trap, as we compute it in Sec. III. Thus, we write the work associated with the quasistatic protocol as $W_{\mathrm{Q}}=W_{\mathrm{H}}+W_{\mathrm{P}}+O\left(b_{\mathrm{P}}^{2}\right)$, where $W_{\mathrm{P}}=\int d k_{\mathrm{P}}\left\langle x^{(0)} x^{(1)}\right\rangle_{\mathrm{SS}}$. The positions $x^{(0)}$ and $x_{0}^{(0)}$ follow the dynamics in Eq. (B2), so that the expression of $x^{(0)}$ is given by Eq. (B5). The positions $x^{(1)}$ and $x_{0}^{(1)}$ follow the coupled set of equations

$$
\begin{aligned}
& \frac{d x^{(1)}}{d t}=-\frac{1}{\tau_{\mathrm{d}}}\left(x^{(1)}-x_{0}^{(1)}\right)-\frac{1}{\tau_{\mathrm{P}}} x^{(1)}-\frac{b_{\mathrm{P}}}{\gamma}\left(x^{(0)}\right)^{3}, \\
& \frac{d x_{0}^{(1)}}{d t}=-\frac{\varepsilon}{\tau_{\mathrm{d}}}\left(x_{0}^{(1)}-x^{(1)}\right),
\end{aligned}
$$

from which we deduce

$$
x^{(1)}(t)=-b_{\mathrm{P}} \int^{t} d t^{\prime} \chi\left(t-t^{\prime}\right)\left(x^{(0)}\right)^{3}\left(t^{\prime}\right) .
$$

We split the correlation function in the definition of $W_{\mathrm{P}}$ in three contributions,

$$
\left\langle x^{(0)} x^{(1)}\right\rangle_{\mathrm{SS}}=-\gamma b_{\mathrm{P}} \lim _{t \rightarrow \infty}\left(C_{1}+C_{2}+C_{3}\right)(t)
$$

where the functions $C_{1}, C_{2}$, and $C_{3}$ read

$$
C_{1}(t)=\iint^{t} \int d u d s \chi(t-u) \chi_{\mathrm{A}}(t-s)\left\langle v_{\mathrm{A}}(s)\left(x^{(0)}\right)^{3}(u)\right\rangle,
$$

$$
C_{2}(t)=\int t d u d s \chi(t-u) \chi_{\mathrm{A}}(t-s) \sqrt{2 \varepsilon D_{\mathrm{T}}}\left\langle\xi_{0}(s)\left(x^{(0)}\right)^{3}(u)\right\rangle,
$$

$$
C_{3}(t)=\iint^{t} d u d s \chi(t-u) \chi(t-s) \sqrt{2 D_{\mathrm{T}}}\left\langle\xi(s)\left(x^{(0)}\right)^{3}(u)\right\rangle .
$$

By using Eq. (B5), we deduce

$$
\begin{aligned}
C_{1}(t)= & \gamma^{3} \int^{t} \int d u d s \int^{u} \iint d u_{1} d u_{2} d u_{3} \chi(t-u) \chi_{\mathrm{A}}(t-s) \\
& \times\left[\chi_{\mathrm{A} 1} \chi_{\mathrm{A} 2} \chi_{\mathrm{A} 3}\left\langle v_{\mathrm{A}}(s) v_{\mathrm{A}}\left(u_{1}\right) v_{\mathrm{A}}\left(u_{2}\right) v_{\mathrm{A}}\left(u_{3}\right)\right\rangle\right. \\
& +6 \varepsilon D_{\mathrm{T}} \chi_{\mathrm{A} 1} \chi_{\mathrm{A} 2} \chi_{\mathrm{A} 3}\left\langle v_{\mathrm{A}}(s) v_{\mathrm{A}}\left(u_{1}\right)\right\rangle\left\langle\xi_{0}\left(u_{2}\right) \xi_{0}\left(u_{3}\right)\right\rangle \\
& \left.+6 D_{\mathrm{T}} \chi_{\mathrm{A} 1} \chi_{2} \chi_{3}\left\langle v_{\mathrm{A}}(s) v_{\mathrm{A}}\left(u_{1}\right)\right\rangle\left\langle\xi\left(u_{2}\right) \xi\left(u_{3}\right)\right\rangle\right]
\end{aligned}
$$

$$
\begin{aligned}
C_{2}(t)= & \gamma^{3} \int^{t} \int d u d s \int^{u} \iint d u_{1} d u_{2} d u_{3} \chi(t-u) \chi_{\mathrm{A}}(t-s) \\
& \times\left[4\left(\varepsilon D_{\mathrm{T}}\right)^{2} \chi_{\mathrm{A} 1} \chi_{\mathrm{A} 2} \chi_{\mathrm{A} 3}\left\langle\xi_{0}(s) \xi_{0}\left(u_{1}\right) \xi_{0}\left(u_{2}\right) \xi_{0}\left(u_{3}\right)\right\rangle\right. \\
& +6 \varepsilon D_{\mathrm{T}} \chi_{\mathrm{A} 1} \chi_{\mathrm{A} 2} \chi_{\mathrm{A} 3}\left\langle\xi_{0}(s) \xi_{0}\left(u_{1}\right)\right\rangle\left\langle v_{\mathrm{A}}\left(u_{2}\right) v_{\mathrm{A}}\left(u_{3}\right)\right\rangle \\
& \left.+12 \varepsilon D_{\mathrm{T}}^{2} \chi_{\mathrm{A} 1} \chi_{2} \chi_{3}\left\langle\xi_{0}(s) \xi_{0}\left(u_{1}\right)\right\rangle\left\langle\xi\left(u_{2}\right) \xi\left(u_{3}\right)\right\rangle\right],
\end{aligned}
$$




$$
\begin{aligned}
C_{3}(t)= & \gamma^{3} \iint d u d s \int^{u} \iint d u_{1} d u_{2} d u_{3} \chi(t-u) \chi(t-s) \\
& \times\left[4 D_{\mathrm{T}}^{2} \chi_{1} \chi_{2} \chi_{3}\left\langle\xi(s) \xi\left(u_{1}\right) \xi\left(u_{2}\right) \xi\left(u_{3}\right)\right\rangle\right. \\
& +6 D_{\mathrm{T}} \chi_{1} \chi_{\mathrm{A} 2} \chi_{\mathrm{A} 3}\left\langle\xi(s) \xi\left(u_{1}\right)\right\rangle\left\langle v_{\mathrm{A}}\left(u_{2}\right) v_{\mathrm{A}}\left(u_{3}\right)\right\rangle \\
& \left.+12 \varepsilon D_{\mathrm{T}}^{2} \chi_{1} \chi_{\mathrm{A} 2} \chi_{3}\left\langle\xi(s) \xi\left(u_{1}\right)\right\rangle\left\langle\xi_{0}\left(u_{2}\right) \xi_{0}\left(u_{3}\right)\right\rangle\right]
\end{aligned}
$$

The non-Gaussianity of the active bursts plays a role in the first term in the bracket of Eq. (B20a). From Eqs. (A3) and (B14), we compute the three contributions of $\left\langle x^{(0)} x^{(1)}\right\rangle_{\mathrm{Ss}}$, and we deduce the expression of this steady-state average to leading order in $\varepsilon$ :

$$
\begin{aligned}
\left\langle x^{(0)} x^{(1)}\right\rangle_{\mathrm{SS}}= & -\left(\frac{T_{\mathrm{A}}}{\varepsilon}\right)^{2} \frac{b_{\mathrm{P}} k^{2} \tau}{k_{\mathrm{P}}\left(k+k_{\mathrm{P}}\right)^{3}\left[k_{\mathrm{P}} \tau+k\left(\tau+\tau_{\mathrm{d}}\right)\right]} \\
& \times\left[2+\left(\frac{k}{k_{\mathrm{P}}}\right)^{2} \frac{5 \tau+2 \tau_{\mathrm{d}}}{2 \tau}+\frac{k}{k_{\mathrm{P}}} \frac{9 \tau+4 \tau_{\mathrm{d}}}{2 \tau}\right] .
\end{aligned}
$$

Finally, the expression of $E_{\mathrm{Q} 1}$ is given by the primitive of the above formula with respect to $k_{\mathrm{P}}$, thus being defined up to a constant.
[1] É. Fodor, M. Guo, N. S. Gov, P. Visco, D. A. Weitz, and F. van Wijland (unpublished).

[2] P. Bursac, G. Lenormand, B. F. M. Oliver, D. A. Weitz, V. Viasnoff, J. P. Butler, and J. J. Fredberg, Nat. Mater. 4, 557 (2005).

[3] T. Toyota, D. A. Head, C. F. Schmidt, and D. Mizuno, Soft Matter 7, 3234 (2011).

[4] B. Stuhrmann, M. Soares e Silva, M. Depken, F. C. MacKintosh, and G. H. Koenderink, Phys. Rev. E 86, 020901 (2012).

[5] C. Wilhelm, Phys. Rev. Lett. 101, 028101 (2008).

[6] D. Robert, T.-H. Nguyen, F. Gallet, and C. Wilhelm, PLoS ONE 5, e10046 (2010).

[7] T. Gisler and D. A. Weitz, Phys. Rev. Lett. 82, 1606 (1999).

[8] M. Yanai, J. P. Butler, T. Suzuki, H. Sasaki, and H. Higuchi, Am. J. Physiol. Cell Physiol. 287, C603 (2004).

[9] N. Desprat, A. Richert, J. Simeon, and A. Asnacios, Biophys. J. 88, 2224 (2005).

[10] M. Balland, N. Desprat, D. Icard, S. Féréol, A. Asnacios, J. Browaeys, S. Hénon, and F. Gallet, Phys. Rev. E 74, 021911 (2006).

[11] D. Mizuno, C. Tardin, C. F. Schmidt, and F. C. MacKintosh, Science 315, 370 (2007).

[12] L. F. Cugliandolo, J. Kurchan, and G. Parisi, J. Phys. I 4, 1641 (1994).

[13] E. Ben-Isaac, Y. K. Park, G. Popescu, F. L. H. Brown, N. S. Gov, and Y. Shokef, Phys. Rev. Lett. 106, 238103 (2011).

[14] J. Prost, J.-F. Joanny, and J. M. R. Parrondo, Phys. Rev. Lett. 103, 090601 (2009).

[15] T. Betz, M. Lenz, J.-F. Joanny, and C. Sykes, Proc. Natl. Acad. Sci. USA 106, 15320 (2009).

[16] F. Gallet, D. Arcizet, P. Bohec, and A. Richert, Soft Matter 5, 2947 (2009).

[17] K. Kanazawa, T. Sagawa, and H. Hayakawa, Phys. Rev. E 90, 012115 (2014).

[18] P. Bohec, F. Gallet, C. Maes, S. Safaverdi, P. Visco, and F. van Wijland, Europhys. Lett. 102, 50005 (2013).

[19] C. Maes, S. Safaverdi, P. Visco, and F. van Wijland, Phys. Rev. E 87, 022125 (2013).

[20] T. Harada and S.-i. Sasa, Phys. Rev. Lett. 95, 130602 (2005).

[21] N. E. Hill, Proc. Phys. Soc. 82, 723 (1963).
[22] V. F. Sears, Proc. Phys. Soc. 86, 953 (1965).

[23] W. T. Coffey and Y. P. Kalmykov, The Langevin Equation: With Applications to Stochastic Problems in Physics, Chemistry and Electrical Engineering, 3rd ed. (World Scientific, Singapore, 2012).

[24] T. G. Mason, Rheol. Acta 39, 371 (2000).

[25] T. G. Mason and D. A. Weitz, Phys. Rev. Lett. 74, 1250 (1995).

[26] K. Kanazawa, T. Sagawa, and H. Hayakawa, Phys. Rev. Lett. 108, 210601 (2012).

[27] K. Kanazawa, T. Sagawa, and H. Hayakawa, Phys. Rev. E 87, 052124 (2013)

[28] M. Guo, A. J. Ehrlicher, M. H. Jensen, M. Renz, J. R. Moore, R. D. Goldman, J. Lippincott-Schwartz, F. C. Mackintosh, and D. A. Weitz, Biophys. J. 105, 1562 (2013).

[29] A. Brut, A. Arakelyan, A. Petrosyan, S. Ciliberto, R. Dillenschneider, and E. Lutz, Nature (London) 483, 187 (2012).

[30] E. Sevick, R. Prabhakar, S. R. Williams, and D. J. Searles, Annu. Rev. Phys. Chem. 59, 603 (2008).

[31] A. Rahman, Phys. Rev. 136, A405 (1964).

[32] K. Sekimoto and S.-i. Sasa, J. Phys. Soc. Jpn. 66, 3326 (1997).

[33] K. Sekimoto, J. Phys. Soc. Jpn. 66, 1234 (1997).

[34] C. W. Gardiner, Handbook of Stochastic Methods (Springer, Berlin, 2004).

[35] F. Gittes, B. Schnurr, P. D. Olmsted, F. C. MacKintosh, and C. F. Schmidt, Phys. Rev. Lett. 79, 3286 (1997).

[36] A. W. C. Lau, B. D. Hoffmann, A. Davies, J. C. Crocker, and T. C. Lubensky, Phys. Rev. Lett. 91, 198101 (2003).

[37] S. Toyabe, H.-R. Jiang, T. Nakamura, Y. Murayama, and M. Sano, Phys. Rev. E 75, 011122 (2007).

[38] B. Lander, J. Mehl, V. Blickle, C. Bechinger, and U. Seifert, Phys. Rev. E 86, 030401 (2012).

[39] M. Baiesi and C. Maes, New J. Phys. 15, 013004 (2013).

[40] D. A. Sivak and G. E. Crooks, Phys. Rev. Lett. 108, 190602 (2012).

[41] T. Hatano and S.-i. Sasa, Phys. Rev. Lett. 86, 3463 (2001).

[42] P. R. Zulkowski, D. A. Sivak, G. E. Crooks, and M. R. DeWeese, Phys. Rev. E 86, 041148 (2012).

[43] E. Barkai, Y. Garini, and R. Metzler, Phys. Today 65, 29 (2012).

[44] F. Hfling and T. Franosch, Rep. Prog. Phys. 76, 046602 (2013). 